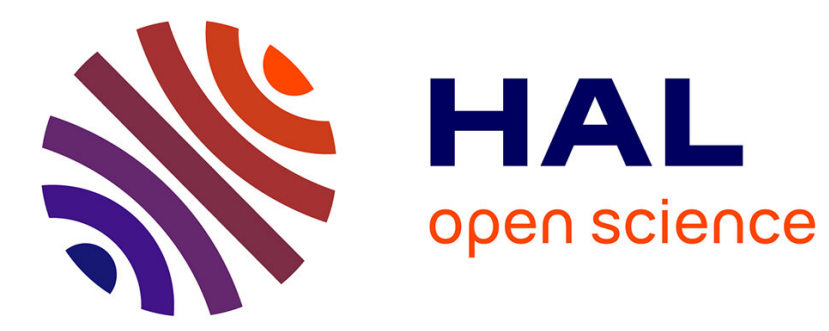

\title{
On existence of oscillations in hybrid systems
}

\author{
Denis Efimov, Wilfrid Perruquetti, Anton Shiriaev
}

\section{To cite this version:}

Denis Efimov, Wilfrid Perruquetti, Anton Shiriaev. On existence of oscillations in hybrid systems. Nonlinear Analysis: Hybrid Systems, 2014, 12, pp.104-116. 10.1016/j.nahs.2013.11.005 . hal00906088

\section{HAL Id: hal-00906088 \\ https://hal.inria.fr/hal-00906088}

Submitted on 19 Nov 2013

HAL is a multi-disciplinary open access archive for the deposit and dissemination of scientific research documents, whether they are published or not. The documents may come from teaching and research institutions in France or abroad, or from public or private research centers.
L'archive ouverte pluridisciplinaire HAL, est destinée au dépôt et à la diffusion de documents scientifiques de niveau recherche, publiés ou non, émanant des établissements d'enseignement et de recherche français ou étrangers, des laboratoires publics ou privés. 


\title{
On existence of oscillations in hybrid systems
}

\author{
Denis Efimov, Wilfrid Perruquetti, Anton Shiriaev
}

\begin{abstract}
The paper extends the notion of oscillations in the sense of Yakubovich to hybrid dynamics. Several sufficient stability and instability conditions for a forward invariant set are presented. The consideration is illustrated by analysis of a model of two-link compass-gait biped robot.
\end{abstract}

\section{Index Terms}

Hybrid systems, oscillations, stability/instability.

\section{INTRODUCTION}

Oscillations constitute one of the main operating modes for many systems in nature or in techniques [1], [2], [3], [4], [5], [6]. In some cases it is required to maintain the oscillations, in other cases the oscillations have to be suppressed [7], [8]. In all cases the conditions of existence of sustained oscillations is of great importance since they allow one to analyze/design a system with desired oscillating properties.

There are many stability theories and definitions of oscillations [8]. Among them in this work we choose one proposed by Prof. Yakubovich almost 40 years ago [9]. This approach is rather generic, and it covers periodical and chaotic oscillations. Contrarily to a pure periodical case, when existence conditions are rather sophisticated [10], the conditions of oscillations in the sense of Yakubovich (Y-oscillations) are simple. For Lurie systems, they are formulated in the frequency domain [9], [11], [12], [4]. For a generic nonlinear system, the conditions of Y-oscillations are given using Lyapunov arguments [13] or applying the homogeneity framework [14]. The main goal of the present paper is to extend the notion of Y-oscillations to hybrid dynamical systems. For this purpose several stability/instability Lyapunov conditions are formulated for hybrid dynamics.

There exist many applications where a system has a hybrid dynamics (continuous and discontinuous) and it is oscillating. The most important one comes from robot locomotion [15], [16]. The phenomenon has a hybrid nature due to impacts occurring when a leg is hitting the ground, and the main operating mode is a periodical oscillation. The design/analysis of robot locomotion [15], [16], [17], [18], [19] or a sliding-mode system [20] as a periodically oscillating system is rather sophisticated. However, relaxing the periodicity requirement and considering Y-oscillations, it is possible to develop more constructive conditions for analysis and design of robot motion.

The problem is introduced and illustrated for a two-link compass-gait biped robot model in Section 2. Some preliminaries are given in Section 3. The main result on conditions of Y-oscillations in hybrid systems is presented in Section 4. Several examples of hybrid oscillating systems are given in Section 5.

The first two authors are with the Non-A project at INRIA - LNE, Parc Scientifique de la Haute Borne, 40 avenue Halley, Bât.A Park Plaza, 59650 Villeneuve d'Ascq, France,\{denis.efimov; wilfrid.perruquetti\}@inria.fr and with LAGIS (UMR-CNRS 8146), Ecole Centrale de Lille, BP 48, Cité Scientifique, 59651 Villeneuve-d'Ascq, France.

The third author is with the Department of Engineering Cybernetics, Norwegian University of Science and Technology, NO-7491 Trondheim, Norway, e-mail: Anton.Shiriaev@itk.ntnu.no. 


\section{MotiVATING EXAMPLE}

Dynamics of a two-link compass-gait biped robot with a control torque applied at the hip (schematically shown in Fig. 1) can be described by the hybrid system [21], [15]:

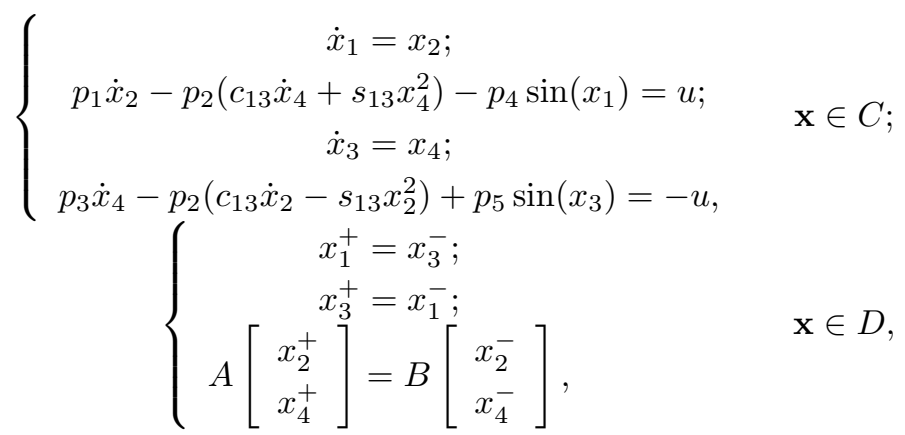

where $\mathbf{x}=\left[\begin{array}{llll}x_{1} & x_{2} & x_{3} & x_{4}\end{array}\right]^{T} \in \mathbb{R}^{4}$ is the state vector of the robot, $u \in \mathbb{R}$ is the control torque; $s_{13}=\sin \left(x_{1}-x_{3}\right), c_{13}=$ $\cos \left(x_{1}-x_{3}\right)$,

$$
A=\left[\begin{array}{cc}
p_{1}-p_{2} c^{-} & p_{3}-p_{2} c^{-} \\
-p_{2} c^{-} & p_{3}
\end{array}\right], B=\left[\begin{array}{cc}
p_{7} c^{-}-p_{6} & -p_{6} \\
-p_{6} & 0
\end{array}\right],
$$

$c^{-}=\cos \left(x_{1}^{-}-x_{3}^{-}\right) ; C=\mathbb{R}^{4}, D=\left\{\mathbf{x} \in \mathbb{R}^{4}: \cos \left(x_{1}+\psi\right)=\cos \left(x_{3}+\psi\right)\right\}$ define the sets with continuous and discrete dynamics respectively; $p_{i}, i=1, \ldots, 7$ are the robot parameters related with its physical counterparts presented in Fig. 1 as $p_{1}=\left(m_{H}+m\right) l^{2}+m a^{2}, p_{2}=m l b, p_{3}=m b^{2}, p_{4}=\left(m_{H} l+m b+m l\right) g, p_{5}=m b g, p_{6}=m a b, p_{7}=m_{H} l^{2}+2 m a l ; \psi$ is the slope of the walking surface. The standard abbreviations

$$
x^{-}=x\left(t^{-}\right)=\lim _{\varepsilon \rightarrow 0} x(t-|\varepsilon|), x^{+}=x\left(t^{+}\right)=\lim _{\varepsilon \rightarrow 0} x(t+|\varepsilon|)
$$

are used to denote the values before and after jumps of a solution. The trajectories of (1) with a unique switch at each passage of $D$ correspond to physical behavior of a compass-gait biped robot.

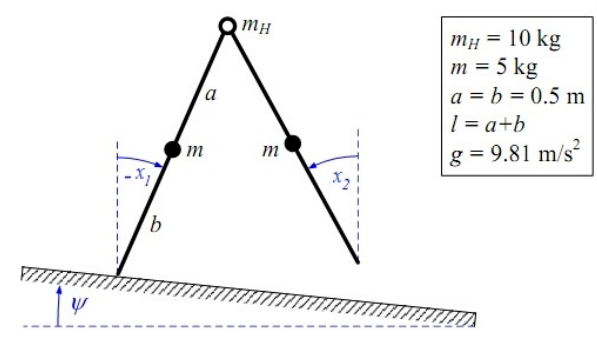

Figure 1. Schematic of the compass-gait biped on a shallow slope

For $\psi=2.87 \pi / 180$ and $u=0$ the system has two (known) nontrivial periodic solutions [15]. If the value of $\psi$ is changed or if it is a time-varying sequence (that corresponds to a walking on an irregular surface), then the conditions of existence of periodical solutions is an open question. That is more, it is hard to address this problem with existing theoretical approaches for a time-varying surface slope.

The tools for detection and stabilization of periodical oscillating modes for a constant $\psi$ are largely reported in the literature (see [21], [15], [16], [17], [18] and references therein). These analysis and design methods are complex and, frequently, only local. The problem complexity is originated by hybrid nature of the dynamics. It is hard to search, analyze and stabilize such a type of behavior. However, if we would skip the requirement that the motion has to be periodical, allowing other types of "oscillating behavior" (that is a natural relaxation for a time-varying $\psi$ ), then existence conditions could be more simple and global depending of course on an alternatively defined oscillation concept. Below such a reduction is demonstrated applying Y-oscillation concept. 


\section{PRELIMINARY RESUltS}

This section has three parts. The first one deals with the hybrid system formalism following [22], [23], [24]. The second part introduces (pre)asymptotic stability definition and its equivalent Lyapunov characterization from [23]. The third part is devoted to sufficient conditions of Lyapunov-type instability for hybrid systems.

In this work, $\mathbb{R}$ denotes the real numbers, $\mathbb{R}_{+}$corresponds to the nonnegative real numbers, $\mathbb{Z}$ and $\mathbb{Z}+$ are stated for integers and nonnegative integers respectively. The symbol $|\cdot|$ denotes an absolute value for a real scalar or Euclidean norm for a vector, $|\mathbf{x}|_{\mathcal{A}}=\inf _{\mathbf{y} \in \mathcal{A}}|\mathbf{y}-\mathbf{x}|$ is the distance from a point $\mathbf{x} \in \mathbb{R}^{n}$ to a set $\mathcal{A} \subset \mathbb{R}^{n}$. A continuous function $\alpha: \mathbb{R}_{+} \rightarrow \mathbb{R}_{+}$ belongs to class $\mathcal{K}$ if $\alpha(0)=0$ and it is strictly increasing, it is from class $\mathcal{K}_{\infty}$ if $\alpha \in \mathcal{K}$ and it is radially unbounded.

\section{A. Hybrid systems}

The considered hybrid system has the following form:

$$
\left\{\begin{array}{cl}
\dot{\mathbf{x}} \in \mathbf{F}(\mathbf{x}) & \mathbf{x} \in C \\
\mathbf{x}^{+} \in \mathbf{G}(\mathbf{x}) & \mathbf{x} \in D
\end{array}\right.
$$

where $\mathrm{x} \in \mathcal{O} \subset \mathbb{R}^{n}$ is the state vector (it may contain continuously changing and discrete or logic-based variables); $C \subset \mathcal{O}$ and $D \subset \mathcal{O}$ are the sets where flow or jumps can occur respectively; the (set-valued) maps $\mathbf{F}: \mathcal{O} \rightrightarrows \mathbb{R}^{n}$ and $\mathbf{G}: \mathcal{O} \rightrightarrows \mathbb{R}^{n}$ determine the continuous and discrete dynamics of the system (2). Following [23] we impose the Standing Assumptions (SAs) on (2):

(SA1) the sets $C \subset \mathcal{O}$ and $D \subset \mathcal{O}$ are relatively closed in $\mathcal{O}$ (i.e. there exist some closed sets $\mathcal{Y}_{C}, \mathcal{Y}_{D}$ such that $D=\mathcal{Y}_{D} \cap \mathcal{O}$ and $\left.C=\mathcal{Y}_{C} \cap \mathcal{O}\right)$;

(SA2) the map $\mathbf{F}: \mathcal{O} \rightrightarrows \mathbb{R}^{n}$ is outer semicontinuous ${ }^{1}$ and locally bounded, $\mathbf{F}(\mathbf{x})$ is nonempty and convex for any $\mathbf{x} \in C$;

(SA3) the map $\mathbf{G}: \mathcal{O} \rightrightarrows \mathbb{R}^{n}$ is outer semicontinuous and locally bounded, $\mathbf{G}(\mathbf{x})$ is nonempty subset of $\mathcal{O}$ for any $\mathbf{x} \in D$.

These assumptions allow one to define solutions of the system (2) on hybrid time domains [22], [23]. A subset $E \subset \mathbb{R}_{+} \times \mathbb{Z}_{+}$ is a compact hybrid time domain if $E=\bigcup_{j=0}^{J-1}\left(\left[t_{j}, t_{j+1}\right], j\right)$ for some finite sequence of times $0 \leq t_{0} \leq \ldots \leq t_{J}$. It is a hybrid time domain if for all $(T, J) \in E, E \cap([0, T] \times\{0,1, \ldots, J\})$ is a compact hybrid time domain. A hybrid arc is a function $\phi: E \rightrightarrows \mathcal{O}$ defined on a hybrid time domain $E$, and such that $\phi(\cdot, j)$ is locally absolutely continuous for each $j$. A hybrid $\operatorname{arc} \phi: \operatorname{dom} \phi \rightrightarrows \mathcal{O}$ is a solution (trajectory) of (2) if $\phi(0,0) \in C \cup D$ and:

$$
\begin{aligned}
& \dot{\phi}(t, j) \in \mathbf{F}[\phi(t, j)] \text { for all } j \in \mathbb{Z}_{+} \text {and almost all } t \in \mathbb{R}_{+} \text {such that }(t, j) \in \operatorname{dom} \phi \text { and } \phi(t, j) \in C ; \\
& \phi(t, j+1) \in \mathbf{G}[\phi(t, j)] \text { for all }(t, j+1) \in \operatorname{dom} \phi \text { and } \phi(t, j) \in D \text {. }
\end{aligned}
$$

The conditions $C \cup D=\mathcal{O}$ guarantees existence of solutions for all initial conditions. A solution $\phi$ to the hybrid system is called nontrivial if $\operatorname{dom} \phi$ contains at least one point different from $(0,0)$; it is called maximal if its domain cannot be extended. A solution is called complete if its domain is unbounded (complete solutions are maximal), let $\mathcal{S}(\mathbf{x})$ denote the set of all maximal solutions for (2) starting from a point $\mathbf{x} \in \mathcal{O}$. The hybrid system (2) is said to be forward complete on $\mathcal{O}$ if for all $\mathbf{x} \in \mathcal{O}$, each $\phi \in \mathcal{S}(\mathbf{x})$ is complete. The hybrid system (2) is said to be forward invariant on a set $\mathcal{A} \subset \mathcal{O}$ if, for all $\mathbf{x} \in \mathcal{A}$, each $\phi \in \mathcal{S}(\mathbf{x})$ is such that $\phi(t, j) \in \mathcal{A}$ for all $(t, j) \in \operatorname{dom} \phi$ (this definition of forward invariance does not need existence of trajectories for all points of $\mathcal{A}$ ). A point $\mathrm{x}_{0}$ is called an equilibrium of (2) if it is forward invariant for the system.

If $G(D) \cap D \neq \emptyset$, then multiple consequent switches or a Zeno-behavior are possible. In many cases (if $D \subset C$, for example) such a behavior does not correspond to physical nature of the considered system, but it is hard to avoid it in the formulation (2) since the sets $C$ and $D$ have to be closed. In addition, if $D \subset C$ then on the intersection $C \cap D$ multiple solutions can be initiated, while frequently in practice only a jump is possible in $D$. Let us define the set of maximal solutions initiated from a point $\mathbf{x} \in \mathcal{O}$ without multiple consequent switches with jumps in $D$ as $\hat{\mathcal{S}}(\mathbf{x})$, i.e. $\hat{\mathcal{S}}(\mathbf{x})=\left\{\phi \in \mathcal{S}(\mathbf{x}): t_{j} \neq t_{j+1}, t_{j+1}=\right.$ $\left.\arg \inf _{t>t_{j}} \phi(t, j) \in D \backslash \Xi,\left(t_{j+1}, j+1\right) \in \operatorname{dom} \phi\right\}$ where the set $\Xi \subset \mathcal{O}$ contains all equilibriums of (2). This set can be empty or, if $G(D) \cap D=\emptyset$, then $\hat{\mathcal{S}}(\mathbf{x})=\mathcal{S}(\mathbf{x})$. Further, talking about a solution $\phi$ of the system (2) we will understand a maximal solution $\phi \in \mathcal{S}(\mathbf{x})$ for some $\mathbf{x} \in \mathcal{O}$, except the restriction $\phi \in \hat{\mathcal{S}}(\mathbf{x})$ is explicitly stated.

\footnotetext{
${ }^{1}$ A set-valued map $\mathbf{F}: \mathcal{O} \rightrightarrows \mathbb{R}^{n}$ is outer semicontinuous at $\overline{\mathbf{x}} \in \mathcal{O}$ if $\lim _{\sup } \sin _{\mathbf{x}} \mathbf{F}(\mathbf{x}) \subset \mathbf{F}(\overline{\mathbf{x}})$, the map $\mathbf{F}: \mathcal{O} \rightrightarrows \mathbb{R}^{n}$ is called outer semicontinuous if it has this property for all $\overline{\mathbf{x}} \in \mathcal{O}$.
} 


\section{B. Stability of hybrid systems}

A continuous function $\omega: \mathcal{O} \rightarrow \mathbb{R}_{+}$is proper on $\mathcal{O}$ if $\omega\left(\mathbf{x}_{i}\right) \rightarrow+\infty$ when $\mathbf{x}_{i}$ converges to a boundary of $\mathcal{O}$ or $\left|\mathbf{x}_{i}\right| \rightarrow+\infty$. For a compact set $\mathcal{A} \subset \mathcal{O}$, the function $\omega$ is a proper indicator for $\mathcal{A}$ on $\mathcal{O}$ if it is proper and $\mathcal{A}=\{\mathbf{x} \in \mathcal{O}: \omega(\mathbf{x})=0\}$.

Definition 1. [23] Let $\mathcal{A} \subset \mathcal{O}$ be compact.

1) $\mathcal{A}$ is prestable for the system (2) if for each $\varepsilon>0$, there exists $\delta>0$ such that any solution $\phi$ with $|\phi(0,0)|_{\mathcal{A}} \leq \delta$ satisfies $|\phi(t, j)|_{\mathcal{A}} \leq \varepsilon$ for all $(t, j) \in \operatorname{dom} \phi$.

2) $\mathcal{A}$ is preattractive for the system (2) if there exists $\delta>0$ such that any solution $\phi$ with $|\phi(0,0)|_{\mathcal{A}} \leq \delta$ is bounded with respect to $\mathcal{O}$, and if it is complete, then $\lim _{t+j \rightarrow+\infty}|\phi(t, j)|_{\mathcal{A}}=0$.

3) $\mathcal{A}$ is preasymptotically stable if it is prestable and preattractive.

4) $\mathcal{A}$ is asymptotically stable if it is preasymptotically stable and there exists $\delta>0$ such that any maximal solution $\phi$ with $|\phi(0,0)|_{\mathcal{A}} \leq \delta$ is complete.

The set of all $\mathrm{x} \in \mathcal{O}$ from which all solutions are bounded with respect to $\mathcal{O}$ and the complete ones converge to $\mathcal{A}$ is called the basin of preattraction and denoted as $\mathcal{O}_{\mathcal{A}}^{a}$. If the conditions of Definition 1 are valid for any $\delta \in \mathbb{R}_{+}$, then the set $\mathcal{A}$ is globally preattractive/preasymptotically or asymptotically stable for (2).

Definition 2. A compact set $\mathcal{A} \subset \mathcal{O}$ is called Non-Zeno (NZ) prestable (preattractive/preasymptotically stable/asymptotically stable) for the system (2) if the corresponding properties of Definition 1 are satisfied for all $\phi \in \hat{\mathcal{S}}$ only.

If for the system (2) the set $\mathcal{A}$ is (pre)asymptotically stable, then it is also NZ (pre)asymptotically stable, the converse is in general false.

Definition 3. Let $\mathcal{X} \subset \mathcal{O}$ be open and $\omega: \mathcal{X} \rightarrow \mathbb{R}_{+}$be continuous. A function $V: \mathcal{X} \rightarrow \mathbb{R}_{+}$is said to be a smooth Lyapunov function for $(\mathcal{X}, \mathbf{F}, \mathbf{G}, C, D, \omega)$ if there exist $\alpha_{1}, \alpha_{2} \in \mathcal{K}_{\infty}$ such that

$$
\alpha_{1}(\omega(\mathbf{x})) \leq V(\mathbf{x}) \leq \alpha_{2}(\omega(\mathbf{x})) \quad \forall \mathbf{x} \in \mathcal{X} .
$$

In this work we will use the following designations:

$$
\begin{aligned}
\operatorname{Sup} D V(\mathbf{x}) & =\sup _{\mathbf{f} \in \mathbf{F}(\mathbf{x})}\langle\nabla V(\mathbf{x}), \mathbf{f}\rangle, \\
\operatorname{Inf} D V(\mathbf{x}) & =\inf _{\mathbf{f} \in \mathbf{F}(\mathbf{x})}\langle\nabla V(\mathbf{x}), \mathbf{f}\rangle ; \\
\operatorname{Sup} V^{+}(\mathbf{x}) & =\sup _{\mathbf{g} \in \mathbf{G}(\mathbf{x}) \cap \mathcal{X}} V(\mathbf{g}), \\
\operatorname{Inf} V^{+}(\mathbf{x}) & =\inf _{\mathbf{g} \in \mathbf{G}(\mathbf{x}) \cap \mathcal{X}} V(\mathbf{g}) .
\end{aligned}
$$

This definition does not impose a restriction on derivative or increment of $V$ since both stability and instability properties will be studied below.

Theorem 1. [23] Let $\mathcal{A}$ be compact and $\mathcal{X}$ be open, $\mathcal{A} \subset \mathcal{X} \subset \mathcal{O}$, and let $\omega$ be a proper indicator for $\mathcal{A}$ on $\mathcal{X}$. The following statements are equivalent for (2):

1) There exists a smooth Lyapunov function $V$ for $(\mathcal{X}, \mathbf{F}, \mathbf{G}, C, D, \omega)$ such that

$$
\begin{aligned}
& \operatorname{Sup} D V(\mathbf{x}) \leq-V(\mathbf{x}) \quad \forall \mathbf{x} \in \mathcal{X} \cap C ; \\
& \operatorname{Sup} V^{+}(\mathbf{x}) \leq e^{-1} V(\mathbf{x}) \quad \forall \mathbf{x} \in \mathcal{X} \cap D
\end{aligned}
$$

and $\mathbf{G}(D \cap \mathcal{X}) \subset \mathcal{X}$.

2) The set $\mathcal{A}$ is preasymptotically stable, its basin of preattraction $\mathcal{O}_{\mathcal{A}}^{a}$ contains $\mathcal{X}$, and $\mathcal{X}$ is forward invariant for (2).

Remark 1. Note that the sufficient part of this theorem holds if the condition (3) is replaced with the following one:

$$
\operatorname{Sup} D V(\mathbf{x}) \leq-\rho[\omega(\mathbf{x})] \quad \forall \mathbf{x} \in \mathcal{X} \cap C ;
$$




$$
\operatorname{Sup} V^{+}(\mathbf{x}) \leq \lambda V(\mathbf{x}) \quad \forall \mathbf{x} \in \mathcal{X} \cap D
$$

for some function $\rho \in \mathcal{K}$ and a constant $\lambda \in[0,1)$. Indeed, in this case $V[\phi(t, j)] \leq \sigma_{1}\left(V\left[\phi\left(t_{j}, j\right)\right]\right) \sigma_{2}\left(e^{t_{j}-t}\right)$ for all $j \in \mathbb{Z}_{+}$ and $t \in\left[t_{j}, t_{j+1}\right]$ such that $(t, j) \in \operatorname{dom} \phi$ and $\phi(t, j) \in C$ for some functions $\sigma_{1}, \sigma_{2} \in \mathcal{K} ;$ and $V[\phi(t, j+1)] \leq \lambda V[\phi(t, j)]$ for all $(t, j+1) \in \operatorname{dom} \phi$ and $\phi(t, j) \in D$. Therefore, according to Definition 1 the system is prestable and preattractive with respect to the set $\mathcal{A}(\mathcal{A}$ is preasymptotically stable).

Remark 2. For the sufficient part of Theorem 1 it is enough to have a continuously differentiable or a Lipschitz continuous function $V$ (the same for the results presented below). For a Lipschitz continuous function $V$ the Dini derivatives have to be used.

Following [24], a more simple for verification sufficient condition can be proposed for NZ preasymptotical stability of $\mathcal{A}$ based on the following assumption.

Assumption 1. For all $\phi \in \hat{\mathcal{S}}$, $\sup _{(t, j) \in \operatorname{dom} \phi} t=+\infty$ iff $\sup _{(t, j) \in \operatorname{dom} \phi} j=+\infty$.

These conditions imply that the hybrid time domains of complete solutions in $\hat{\mathcal{S}}$ are unbounded with respect to both $t$ and $j$. This assumption excludes from consideration a case with a finite number of jumps and the Zeno-behavior. The reasons for this condition introduction are the following. If the number of switches is finite for a complete solution $\phi \in \mathcal{S}$, then the asymptotic transients are predefined by the continuous dynamics only. The Zeno-behavior is rather usual in hybrid models, but frequently it has no physical meaning (like in the compass-gait biped robot or the bouncing ball [23], for instance) since multiple impacts are possible in an equilibrium only.

Theorem 2. Let $\mathcal{A}$ be compact and $\mathcal{X}$ be open, $\mathcal{A} \subset \mathcal{X} \subset \mathcal{O}$, and let $\omega$ be a proper indicator for $\mathcal{A}$ on $\mathcal{X}$. Let Assumption 1 be satisfied and there exist a continuously differentiable Lyapunov function $V$ for $(\mathcal{X}, \mathbf{F}, \mathbf{G}, C, D, \omega)$ such that

$$
\operatorname{Sup} D V(\mathbf{x}) \leq 0 \quad \forall \mathbf{x} \in \mathcal{X} \cap C
$$

$\mathbf{G}(D \cap \mathcal{X}) \subset \mathcal{X}$ and for any solution $\phi \in \hat{\mathcal{S}}$ for all $\left(t_{j+1}, j+1\right) \in$ dom $\phi$ such that $\phi\left(t_{j}, j\right) \in \mathcal{X}$ and $\phi\left(t_{j+1}, j+1\right) \in \mathcal{X}$ for some $\alpha \in \mathcal{K}$

$$
\frac{V\left[\phi\left(t_{j+1}, j+1\right)\right]-V\left[\phi\left(t_{j}, j\right)\right]}{t_{j+1}-t_{j}} \leq-\alpha\left(V\left[\phi\left(t_{j+1}, j+1\right)\right]\right) .
$$

Then the set $\mathcal{A}$ is $N Z$ preasymptotically stable, its basin of preattraction $\mathcal{O}_{\mathcal{A}}^{a}$ contains $\mathcal{X}$ and $\mathcal{X}$ is forward invariant for $\phi \in \hat{\mathcal{S}}$ of $(2)$.

Proof: Note that $V[\phi(t, j)] \leq V\left[\phi\left(t_{j}, j\right)\right]$ for all $j \in \mathbb{Z}_{+}$and $t \in\left[t_{j}, t_{j+1}\right]$ such that $(t, j) \in \operatorname{dom} \phi$ and $\phi(t, j) \in C$ (this relation follows from (5)). According to (6), in addition, $V\left[\phi\left(t_{j+1}, j+1\right)\right] \leq V\left[\phi\left(t_{j}, j\right)\right]$ for all $\left(t_{j+1}, j+1\right) \in \operatorname{dom} \phi$. Combining these facts, since $V\left[\phi\left(t_{j+1}, j\right)\right] \leq V\left[\phi\left(t_{j}, j\right)\right]$, we have that $V[\phi(t, j)] \leq V[\phi(0,0)]$ for all $(t, j) \in \operatorname{dom} \phi, \phi \in \hat{\mathcal{S}}$ and the set $\mathcal{A}$ is NZ prestable for the system (2) and it is forward complete. Define $V^{j}=V\left[\phi\left(t_{j}, j\right)\right]$ to simplify the notation, then

$$
\begin{aligned}
\Delta V^{j}=V^{j+1}-V^{j} & \leq-\left(t_{j+1}-t_{j}\right) \alpha\left(V^{j+1}\right) \\
& \leq-\left(t_{j+1}-t_{j}\right) \alpha\left(V^{N+1}\right)
\end{aligned}
$$

for all $\left(t_{N}, N\right) \in \operatorname{dom} \phi$ and $j \leq N$ (as it was noted above the sequence $V^{j}$ is not increasing). In addition, $t_{j+1}-t_{j}>0$ since $\phi \in \hat{\mathcal{S}}$. Thus

$$
V^{N+1}-V^{0}=\sum_{j=0}^{N} \Delta V^{j} \leq-\left(t_{N+1}-t_{0}\right) \alpha\left(V^{N+1}\right)
$$

and

$$
\alpha\left(V^{N+1}\right) \leq \frac{V^{0}-V^{N+1}}{t_{N+1}-t_{0}} \leq \frac{V^{0}}{t_{N+1}-t_{0}} .
$$

Under Assumption 1 there is an infinite number of switches and $t_{N+1} \rightarrow+\infty$, consequently $\lim _{t+j \rightarrow+\infty} V[\phi(t, j)]=0$ or equivalently ( $V$ is a Lyapunov function) $\lim _{t+j \rightarrow+\infty}|\phi(t, j)|_{\mathcal{A}}=0$, therefore $\mathcal{A}$ is $\mathrm{NZ}$ preattractive for the system (2). 
Despite of the conditions of Theorem 2 include the inequality (6) in the time domain for $\phi \in \hat{\mathcal{S}}$, in some cases it is more simple to apply Theorem 2 than Theorem 1 (that we will show later on the examples). Note that without the condition $\operatorname{Sup} V^{+}(\mathbf{x}) \leq V(\mathbf{x}) \quad \forall \mathbf{x} \in \mathcal{X} \cap D$ the function $V$ decreasing may be non-monotone, i.e. $V\left[\phi\left(t_{j+1}, j+1\right)\right]>V\left[\phi\left(t_{j+1}, j\right)\right]$ for some $\left(t_{j+1}, j+1\right) \in \operatorname{dom} \phi$ and $\phi\left(t_{j+1}, j\right) \in D$. In another case $V$ may be decreasing on jumps in $D$, but not necessarily strictly decreasing for the continuous flow in $C$.

\section{Instability of hybrid systems}

This section contains a definition of instability (similarly to [25], [24]) and the corresponding sufficient Lyapunov-type conditions.

Definition 4. Let $\mathcal{U} \subset \mathcal{O}$ be compact.

1) $\mathcal{U}$ is preunstable for the system (2) if it is not prestable in the sense of Definition 1, i.e. there exists $\varepsilon>0$ such that for each $\delta>0$ there is a solution $\phi$ with $|\phi(0,0)|_{\mathcal{U}} \leq \delta$ that satisfies $|\phi(t, j)|_{\mathcal{U}}>\varepsilon$ for some $(t, j) \in \operatorname{dom} \phi$.

2) $\mathcal{U}$ is prerepulsive for the system (2) if there is some $\bar{\delta}>0$ such that for any $0<\delta \leq \bar{\delta}$ there exists a solution $\phi$ with $|\phi(0,0)|_{\mathcal{U}} \leq \delta$ for which there are some $\left(t^{\prime}, j^{\prime}\right) \in \operatorname{dom} \phi$ such that the property $|\phi(t, j)|_{\mathcal{U}}>\bar{\delta}$ holds for all $(t, j) \in \operatorname{dom} \phi$ with $t \geq t^{\prime}, j \geq j^{\prime}$.

3) $\mathcal{U}$ is strictly prerepulsive for the system (2) if for some $\bar{\delta}>0$ and any solution $\phi$ with $|\phi(0,0)|_{\mathcal{U}} \leq \bar{\delta}$ there exist some $\left(t^{\prime}, j^{\prime}\right) \in \operatorname{dom} \phi$ such that the property $|\phi(t, j)|_{\mathcal{U}}>\bar{\delta}$ holds for all $(t, j) \in \operatorname{dom} \phi$ with $t \geq t^{\prime}, j \geq j^{\prime}$.

As in the continuous case [26], the hybrid system (2) can be preattractive with respect to a set $\mathcal{A}$ and preunstable with respect to this set simultaneously. If the set $\mathcal{U}$ is prerepulsive for the system (2), then it is preunstable, but this set still can be attractive for a some subset of initial states in a vicinity of $\mathcal{U}$. If it is strictly prerepulsive, then all existent solutions exit a neighborhood of $\mathcal{U}$. The domain of prerepulsion $\mathcal{O}_{\mathcal{U}}^{r}$ contains $\mathbf{x} \in \mathcal{O}$ such that $\phi(0,0)=\mathbf{x}$ and $|\phi(t, j)|_{\mathcal{U}}>\bar{\delta}$ for all $(t, j) \in \operatorname{dom} \phi$ with $t \geq t^{\prime}, j \geq j^{\prime}\left(\left(t^{\prime}, j^{\prime}\right) \in \operatorname{dom} \phi\right)$.

Definition 5. A compact set $\mathcal{U} \subset \mathcal{O}$ is called $\mathrm{NZ}$ preunstable (prerepulsive/strictly prerepulsive) for the system (2) if the corresponding properties of Definition 4 are satisfied for $\phi \in \hat{\mathcal{S}}$ only.

If $\mathcal{U}$ is NZ preunstable/prerepulsive for (2), then it is also preunstable/prerepulsive. If $\mathcal{U}$ is strictly prerepulsive, then it is also NZ strictly prerepulsive.

Theorem 3. Let $\mathcal{U} \subset \mathcal{X}$ be compact and $\mathcal{X} \subset \mathcal{O}$ be open, $\mathbf{G}(D \cap \mathcal{X}) \subset \mathcal{X}$. Let a continuous function $\omega: \mathcal{X} \rightarrow \mathbb{R}_{+}$be a proper indicator for $\mathcal{U}$ on $\mathcal{X}$. Let also each $\phi \in \mathcal{S}$ with $\phi(0,0) \in \mathcal{X}$ be nontrivial.

1) The set $\mathcal{U}$ is strictly prerepulsive for the system (2), its domain of prerepulsion $\mathcal{O}_{\mathcal{U}}^{r}$ contains $\mathcal{X}$, if there exists a continuously differentiable Lyapunov function $U: \mathcal{X} \rightarrow \mathbb{R}_{+}$for $(\mathcal{X}, \mathbf{F}, \mathbf{G}, C, D, \omega)$ such that for some function $\rho \in \mathcal{K}$ and a constant $\lambda \in[1,+\infty)$

$$
\begin{aligned}
\operatorname{Inf} D U(\mathbf{x}) & \geq \rho[\omega(\mathbf{x})] \quad \forall \mathbf{x} \in \mathcal{X} \cap C ; \\
\operatorname{Inf} U^{+}(\mathbf{x}) & \geq \lambda U(\mathbf{x}) \quad \forall \mathbf{x} \in \mathcal{X} \cap D .
\end{aligned}
$$

2) Let Assumption 1 be satisfied. The set $\mathcal{U}$ is $N Z$ strictly prerepulsive for the system (2), its domain of prerepulsion $\mathcal{O}_{\mathcal{U}}^{r}$ contains $\mathcal{X}$ for $\phi \in \hat{\mathcal{S}}$, if there exists a continuously differentiable Lyapunov function $U: \mathcal{X} \rightarrow \mathbb{R}_{+}$for $(\mathcal{X}, \mathbf{F}, \mathbf{G}, C, D, \omega)$ such that for any solution $\phi \in \hat{\mathcal{S}}$ for all $\left(t_{j+1}, j+1\right) \in \operatorname{dom} \phi$ such that $\phi\left(t_{j}, j\right) \in \mathcal{X}$ and $\phi\left(t_{j+1}, j+1\right) \in \mathcal{X}$ for some $\alpha \in \mathcal{K}$

$$
\frac{U\left[\phi\left(t_{j+1}, j+1\right)\right]-U\left[\phi\left(t_{j}, j\right)\right]}{t_{j+1}-t_{j}} \geq \alpha\left(U\left[\phi\left(t_{j}, j\right)\right]\right)
$$

and

$$
\operatorname{Inf} D U(\mathbf{x}) \geq 0 \quad \forall \mathbf{x} \in \mathcal{X} \cap C
$$


Proof: In the first case $U[\phi(t, j)] \geq \sigma_{1}\left(U\left[\phi\left(t_{j}, j\right)\right]\right) \sigma_{2}\left(e^{t-t_{j}}\right)$ for all $j \in \mathbb{Z}_{+}$and $t \in\left[t_{j}, t_{j+1}\right]$ such that $(t, j) \in \operatorname{dom} \phi$ and $\phi(t, j) \in C$ for some functions $\sigma_{1}, \sigma_{2} \in \mathcal{K}$; and $U[\phi(t, j+1)] \geq \lambda U[\phi(t, j)]$ for all $(t, j+1) \in \operatorname{dom} \phi$ and $\phi(t, j) \in D$. Therefore, $U[\phi(t, j)]$ is a strictly increasing function of $(t, j) \in \operatorname{dom} \phi$ while $\phi(t, j) \in \mathcal{X}$. By Definition 4 the system (2) is preunstable and prerepulsive with respect to the set $\mathcal{U}$. The domain of prerepulsion $\mathcal{O}_{\mathcal{U}}^{r}$ contains $\mathcal{X}$.

In the second case, from (9), $U[\phi(t, j)] \geq U\left[\phi\left(t_{j}, j\right)\right]$ for all $j \in \mathbb{Z}_{+}$and $t \in\left[t_{j}, t_{j+1}\right]$ such that $(t, j) \in \operatorname{dom} \phi$ and $\phi(t, j) \in C$, and $U\left[\phi\left(t_{j+1}, j+1\right)\right] \geq U\left[\phi\left(t_{j}, j\right)\right]$ for all $\left(t_{j+1}, j+1\right) \in \operatorname{dom} \phi$ in accordance with (8). Consequently,

$$
\begin{aligned}
\Delta U^{j}=U^{j+1}-U^{j} & \geq\left[t_{j+1}-t_{j}\right] \alpha\left(U^{j}\right) \\
& \geq\left[t_{j+1}-t_{j}\right] \alpha\left(U^{0}\right)
\end{aligned}
$$

for all $\left(t_{j+1}, j+1\right) \in \operatorname{dom} \phi$ since the sequence $U^{j}=U\left[\phi\left(t_{j}, j\right)\right]$ is not decreasing $\left(t_{j+1}>t_{j}\right.$ since $\left.\phi \in \hat{\mathcal{S}}\right)$. Finally we obtain

$$
U^{j+1}-U^{0}=\sum_{i=0}^{j} \Delta U^{i} \geq\left[t_{j+1}-t_{0}\right] \alpha\left(U^{0}\right)
$$

and

$$
U^{j+1} \geq U^{0}+\left[t_{j+1}-t_{0}\right] \alpha\left(U^{0}\right) .
$$

Since under Assumption 1 for a complete solution $\phi \in \hat{\mathcal{S}}$, we have $t_{j+1} \rightarrow+\infty$ with $j \rightarrow+\infty$, then the sequence $U^{j}$ is unbounded. If the solution $\phi$ is not complete, then the trajectory escapes to infinity in a finite time, hence $U$ is again unbounded. A solution $\phi$ may also leave $\mathcal{X}$ in a finite time. Then by Definition 4 the system (2) is preunstable and prerepulsive with respect to the set $\mathcal{U}$ (again $\mathcal{X} \subset \mathcal{O}_{\mathcal{U}}^{r}$ ).

Note that the condition (8) is sufficient to prove that the set $\mathcal{U}$ is preunstable for the system (2). Contrarily the condition (7), the conditions (8), (9) do not restrict the Lyapunov function $U$ behavior after switches (it may decrease on $D$ ).

\section{OSCILLATION CONDITIONS}

Extending the results from [13], [9], the definition of Y-oscillations for hybrid systems can be as follows (recall, a function $g: \mathbb{R}^{n} \rightarrow \mathbb{R}$ is called monotone if the condition $x_{1} \leq x_{1}^{\prime}, \ldots, x_{n} \leq x_{n}^{\prime}$ implies that everywhere either $g\left(x_{1}, \ldots, x_{n}\right) \leq$ $g\left(x_{1}^{\prime}, \ldots, x_{n}^{\prime}\right)$ or $\left.g\left(x_{1}, \ldots, x_{n}\right) \geq g\left(x_{1}^{\prime}, \ldots, x_{n}^{\prime}\right)\right)$.

Definition 6. [9], [13] A complete solution $\phi \in \mathcal{S}(\mathbf{x})$ with $\mathbf{x} \in \mathcal{O}$ of the system (2) is called $\left[\pi^{-}, \pi^{+}\right]$-oscillation with respect to the output $\psi=\eta(\mathbf{x})$ (where $\eta: \mathcal{O} \rightarrow \mathbb{R}$ is a continuous monotone function) if

$$
\begin{gathered}
\varliminf_{t+j \rightarrow+\infty} \eta[\phi(t, j)]=\pi^{-} ; \varlimsup_{t+j \rightarrow+\infty} \eta[\phi(t, j)]=\pi^{+} ; \\
-\infty<\pi^{-}<\pi^{+}<+\infty .
\end{gathered}
$$

A complete solution $\phi \in \mathcal{S}(\mathbf{x})$ with $\mathbf{x} \in \mathcal{O}$ of the system (2) is called oscillating, if there exist some output $\psi$ and constants $\pi^{-}, \pi^{+}$such that $\phi$ is $\left[\pi^{-}, \pi^{+}\right]$-oscillation with respect to the output $\psi$. A forward complete system (2) is called oscillatory, if for almost all $\mathbf{x} \in \mathcal{O}$ the solutions $\phi \in \mathcal{S}(\mathbf{x})$ of the system are oscillating. The oscillatory system (2) is called uniformly oscillatory, if for almost all $\mathbf{x} \in \mathcal{O}$ for corresponding solutions $\phi \in \mathcal{S}(\mathbf{x})$ there exist the output $\psi$ and the constants $\pi^{-}, \pi^{+}$ not depending on initial conditions.

In other words the solution $\phi$ is oscillating if the output $\psi(t, j)=\eta[\phi(t, j)]$ is asymptotically bounded and there is no single limit value of $\psi(t, j)$ for $t+j \rightarrow+\infty$. The term "almost all solutions" is used to emphasize that generally the system (2) has a nonempty set of equilibrium points, thus there exists a set of initial conditions with zero measure such that the corresponding solutions are not oscillating. The notion of oscillations in the sense of Yakubovich is rather generic including periodical oscillations (limit cycles), quasi-periodical, recurrent and chaotic trajectories. The oscillating trajectories could be repelling being oscillating. The trajectories could also be unbounded, it is required to find a function of the state vector $\eta$, that is bounded and admits certain requirements introduced in Definition 6. 
Theorem 4. Let $\mathcal{A} \subset \mathcal{X}_{\mathcal{A}}$ and $\mathcal{U} \subset \mathcal{X}_{\mathcal{U}} \subset \mathcal{A}$ be compact and $\mathcal{X}=\mathcal{X}_{\mathcal{A}} \cup \mathcal{X}_{\mathcal{U}} \subset \mathcal{O}$ be open. Let continuous functions $\omega_{\mathcal{A}}: \mathcal{X} \rightarrow \mathbb{R}_{+}$, $\omega_{\mathcal{U}}: \mathcal{X} \rightarrow \mathbb{R}_{+}$be proper indicators for $\mathcal{A}$ and $\mathcal{U}$ on $\mathcal{X}$ respectively. Let $\mathbf{G}(D \cap \mathcal{X}) \subset \mathcal{X}$, each $\phi \in \mathcal{S}$ with $\phi(0,0) \in \mathcal{X}$ be nontrivial and the system (2) have two continuously differentiable Lyapunov functions $V$ and $U$ such that for all $\mathrm{x} \in \mathcal{X}:$

$$
\begin{aligned}
& v_{1}\left[\omega_{\mathcal{A}}(\mathbf{x})\right] \leq V(\mathbf{x}) \leq v_{2}\left[\omega_{\mathcal{A}}(\mathbf{x})\right], \\
& v_{3}\left[\omega_{\mathcal{U}}(\mathbf{x})\right] \leq U(\mathbf{x}) \leq v_{4}\left[\omega_{\mathcal{U}}(\mathbf{x})\right],
\end{aligned}
$$

$v_{1}, v_{2}, v_{3}, v_{4} \in \mathcal{K}_{\infty}$ and

1) the conditions (4) or (5), (6) with Assumption 1 are valid for the function $V$ on $\mathcal{X}_{\mathcal{A}}$;

2) the conditions (7) or (8), (9) with Assumption 1 are valid for the function $U$ on $\mathcal{X}_{\mathcal{U}}$;

3) $\Omega \cap \Xi=\emptyset$, where $\Omega=\mathcal{A} \cap \mathcal{X}_{\mathcal{U}}$ and $\Xi \subset \mathbb{R}^{n}$ is a set with zero Lebesgue measure containing all equilibriums of the system.

Then the system (2) has an oscillating solution.

Proof: According to the results of theorems 1, 2 and 3, the set $\mathcal{A}$ is NZ preasymptotically stable (its basin of preattraction $\mathcal{O}_{\mathcal{A}}^{a}$ contains $\mathcal{X}_{\mathcal{A}}$, and $\mathcal{X}_{\mathcal{A}}$ is forward invariant for (2)) and the set $\mathcal{U}$ is $\mathrm{NZ}$ strictly prerepulsive for the system (2), its domain of prerepulsion $\mathcal{O}_{\mathcal{U}}^{r}$ contains $\mathcal{X}_{\mathcal{U}}$. Thus in $\mathcal{X}_{\mathcal{A}}$ all solutions $\phi \in \hat{\mathcal{S}}(\mathrm{x})$ approach the set $\mathcal{A}$, while inside this set there is a repulsing subset $\mathcal{U}$ with the repulsion region $\mathcal{X}_{\mathcal{U}}$, then all $\phi \in \hat{\mathcal{S}}(\mathbf{x})$ have to approach the intersection $\Omega=\mathcal{A} \cap \mathcal{X}_{\mathcal{U}}$, a forward invariant compact set for the system (2) for $\phi \in \hat{\mathcal{S}}(\mathbf{x})$. The set $\Omega$ does not contain the system equilibriums by the theorem conditions. Consider a solution $\phi \in \hat{\mathcal{S}}(\mathrm{x})$ with $\mathrm{x} \in \Omega$ of the system (2) (it is complete by the consideration above). Then there exists $\psi=\eta(\mathbf{x})=x_{i}$ for some $1 \leq i \leq n$ (the $i^{\text {th }}$ coordinate of the system) such that the solution $\phi$ is oscillating with respect to this output $\psi$. Assume it is not, it implies that $\underline{\lim }_{t+j \rightarrow+\infty} \phi_{i}(t, j)=\varlimsup_{t+j \rightarrow+\infty} \phi_{i}(t, j)$ for all $1 \leq i \leq n$ and that $\phi$ converges to an equilibrium point. Since there is no equilibriums in $\Omega$, there is such an index $i$ with $-\infty<\underline{\lim }_{t+j \rightarrow+\infty} \phi_{i}(t, j)<\varlimsup_{\lim }^{t+j \rightarrow+\infty} \phi_{i}(t, j)<+\infty$ and the solution $\phi$ is oscillating.

Remark 3. If the conditions (4) and (7) are satisfied, then the system (2) is oscillating.

Remark 4. The obtained in Theorem 4 conditions extend the results of the work [27], obtained for a linear system with a sliding-mode feedback (in that case the solutions are absolutely continuous), on a more general case.

In the next section we will show several examples of this theorem application.

\section{EXAMPLES}

In this section we will consider three examples, in all cases $D \subset C$.

\section{A. A planar hybrid system}

Consider the following planar hybrid system:

$$
\mathbf{F}(\mathbf{x})=\left[\begin{array}{c}
(1-|\mathbf{x}|) x_{1}+x_{2} \\
(1-|\mathbf{x}|) x_{2}-x_{1}
\end{array}\right], \mathbf{G}(\mathbf{x})=\left[\begin{array}{c}
x_{2} \\
x_{1}
\end{array}\right],
$$

where $\mathbf{x}=\left[x_{1} x_{2}\right]^{T}, \mathcal{O}=\mathbb{R}^{2}, D=\left\{\mathbf{x} \in \mathcal{O}: x_{1}+k x_{2}=0\right\}, C=\mathcal{O}$ and $k \in \mathbb{R} \backslash\{-1,1\}$ is a parameter.

The system (10) has the single equilibrium at the origin for $k \neq \pm 1(\Xi=\{0\})$. Next, it is easy to verify that the Lyapunov function $W=|\mathbf{x}|^{2}$ for the continuous dynamics $\mathbf{F}$ has the derivative $\dot{W}=2(1-\sqrt{W}) W$ for all $\mathbf{x} \in \mathbb{R}^{2}$, then $W\left(t, t_{0}, W_{0}\right)=\left[1-\left(1-W_{0}^{-0.5}\right) e^{t_{0}-t}\right]^{-2}$ for any initial condition $W\left(t_{0}\right)=W_{0}$, which according to [13], [14] implies existence of oscillating trajectories into the set $W(\mathbf{x})=1$ (for $\dot{\mathbf{x}}=\mathbf{F}(\mathbf{x})$ ) and forward completeness of (10). To prove that for any initial condition $\mathbf{x}\left(t^{\prime}, j\right) \in \mathcal{O}, t^{\prime} \in \mathbb{R}_{+}, j \in \mathbb{Z}_{+}$for solutions of the system (10) always there exists a time instant $t^{\prime \prime} \geq t^{\prime}$ such that $\mathbf{x}\left(t^{\prime \prime}, j\right) \in D$ assume that it is not, then from the expression of $W\left(t, t_{0}, W_{0}\right)$ given above the trajectories of the continuous dynamics converge to the set where $W(\mathbf{x})=1$ and the continuous dynamics is reduced to a linear oscillator, thus $t^{\prime \prime}$ exists and Assumption 1 is satisfied. 
For the chosen $\mathbf{G}$ we have that $W\left(\mathbf{x}^{+}\right)=W(\mathbf{x})$ for all $\mathbf{x} \in D$, therefore the value of $W$ is not changing for the discrete dynamics. In addition, for $k \neq \pm 1$ the set $D \backslash\{0\}$ is not invariant for (10), therefore for almost all instants of time the behavior of $W$ is predefined by the continuous dynamics.

To apply Theorem 4 we can choose the origin as the set $\mathcal{U}, U(\mathbf{x})=W(\mathbf{x})$ and $\mathcal{X}_{\mathcal{U}}=\{\mathbf{x} \in \mathcal{O}:|\mathbf{x}|<1\}$, then $U(t, j)$ is a strictly increasing function on $\mathcal{X}_{\mathcal{U}}$ and for any solution $\phi \in \hat{\mathcal{S}}$ of (10) with $\phi(0,0) \in \mathcal{X}_{\mathcal{U}}$ we have $U\left[\phi\left(t_{j+1}, j+1\right)\right]-$ $U\left[\phi\left(t_{j}, j\right)\right] \geq\left(t_{j+1}-t_{j}\right) 2\left(1-\sqrt{U\left[\phi\left(t_{j}, j\right)\right]}\right) U\left[\phi\left(t_{j}, j\right)\right]$ for all $\left(t_{j}, j\right) \in \operatorname{dom} \phi$ while $\phi$ rests in $\mathcal{X}_{\mathcal{U}}$, i.e. the conditions of Theorem 3 are satisfied.

Let us select the set $\mathcal{A}=\{\mathrm{x} \in \mathcal{O}:|\mathbf{x}| \leq 1\}$, then $\mathcal{X}_{\mathcal{A}}=\mathcal{O} \backslash \mathcal{A}$. Using the function $W$ we can prove that this $\mathcal{A}$ is NZ preasymptotically stable for (10). Indeed, for any solution $\phi \in \hat{\mathcal{S}}$ of (10) with $\phi(0,0) \in \mathcal{X}_{\mathcal{A}}$ we have $W\left[\phi\left(t_{j+1}, j+1\right)\right]-$ $W\left[\phi\left(t_{j}, j\right)\right] \leq\left(t_{j+1}-t_{j}\right) 2\left(1-\sqrt{W\left[\phi\left(t_{j+1}, j+1\right)\right]}\right) W\left[\phi\left(t_{j+1}, j+1\right)\right]$ for all $\left(t_{j}, j\right) \in \operatorname{dom} \phi$ while $\phi$ rests in $\mathcal{X}_{\mathcal{A}}$. Select $V(\mathbf{x})=\max \{0, W(\mathbf{x})-1\}$, then outside of the set $\mathcal{A}$ we have $V\left[\phi\left(t_{j+1}, j+1\right)\right]-V\left[\phi\left(t_{j}, j\right)\right] \leq-\left(t_{j+1}-t_{j}\right) \alpha\left\{V\left[\phi\left(t_{j+1}, j+1\right)\right]\right\}$ for $\alpha(s)=-(1-\sqrt{1+s})(1+s) \in \mathcal{K}$ and all conditions of Theorem 2 are verified.

The set $\Omega=\{\mathbf{x} \in \mathcal{O}:|\mathbf{x}|=1\}$ does not contain the equilibrium of (10). Therefore, all conditions of Theorem 4 are satisfied and the system (10) has an oscillating solution.

The system trajectories for different values of $k$ are shown in Fig. 2. As we can deduce from these results of simulation, the system stay oscillating for all values of $k$, but the types of the oscillating trajectories differ significantly (there exist two disjoint oscillating subsets in $\Omega$ for $k=|1.5|$ and a discontinuous limit cycle for $k=|0.5|$ ).
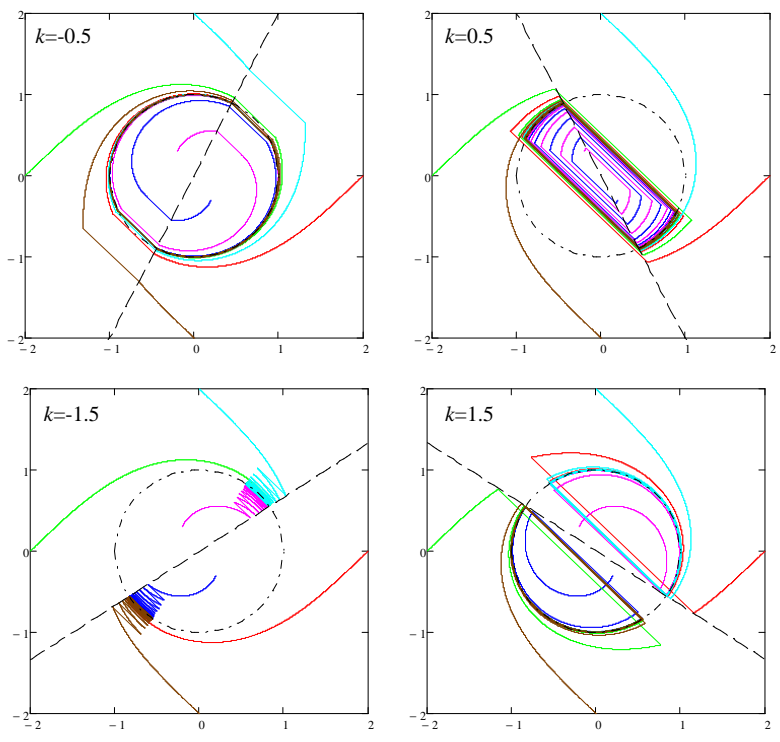

Figure 2. The hybrid system (10) trajectories $\left(x_{1}, x_{2}\right)$ for $k \in\{-0.5,0.5,-1.5,1.5\}$

\section{B. A third order hybrid system}

Another example is a third order hybrid system:

$$
\begin{aligned}
\mathbf{F}(\mathbf{x}) & =\left\{\begin{array}{c}
\mathbf{A}_{1} \mathbf{x} \text { if }|\mathbf{x}|<1 \\
\mathbf{A}_{2} \mathbf{x} \text { if }|\mathbf{x}| \geq 1
\end{array}, \mathbf{G}(\mathbf{x})=\varphi(|\mathbf{x}|)\left[\begin{array}{l}
x_{2} \\
x_{1} \\
x_{3}
\end{array}\right],\right. \\
\mathbf{A}_{1} & =\left[\begin{array}{ccc}
1 & 1 & 0 \\
-1 & 1 & 1 \\
0 & -1 & 1
\end{array}\right], \mathbf{A}_{2}=\left[\begin{array}{ccc}
0 & 1 & 0 \\
-1 & 0 & 1 \\
0 & -1 & 0
\end{array}\right],
\end{aligned}
$$


where $\mathbf{x}=\left[x_{1} x_{2} x_{3}\right]^{T}, \mathcal{O}=\mathbb{R}^{3}, D=\left\{\mathbf{x} \in \mathcal{O}: x_{1}+k x_{2}=x_{3}\right\}$ with $|k| \neq 1, C=\mathcal{O}$ and

$$
\varphi(|\mathbf{x}|)=\left\{\begin{array}{cc}
1 & \text { if }|\mathbf{x}| \leq 1 \\
\frac{4}{3+|\mathbf{x}|} & \text { if }|\mathbf{x}|>1
\end{array}\right. \text {. }
$$

The set $D$ is not invariant for the continuous dynamics for $|k| \neq 1$, the system (11) has the single equilibrium ( $\Xi=\{0\}$ ). The matrix $\mathbf{A}_{1}$ has all eigenvalues with a positive real part and the matrix $\mathbf{A}_{2}$ has eigenvalues with zero real part (two with non-zero imaginary parts). For the continuous dynamics $\mathbf{F}$ consider a Lyapunov function $W(\mathbf{x})=|\mathbf{x}|^{2}$, then

$$
\dot{W}=\left\{\begin{array}{c}
2 W \text { if } W<1 \\
0 \text { if } W \geq 1
\end{array} .\right.
$$

Therefore, for any initial condition $\mathbf{x}\left(t^{\prime}, j\right) \in \mathcal{O}, t^{\prime} \in \mathbb{R}_{+}, j \in \mathbb{Z}_{+}$for solutions of the system (11) always there exists a time instant $t^{\prime \prime} \geq t^{\prime}$ such that $\mathbf{x}\left(t^{\prime \prime}, j\right) \in D$. Indeed, assume that it is not, then from the above expression of $\dot{W}$, for $W \geq 1$ the system $\dot{\mathbf{x}}=\mathbf{F}(\mathbf{x})$ is purely oscillating and always there exists a crossing of $D$ for $|k| \neq 1$, for $W<1$ we have $W(t)=W\left(t^{\prime}\right) e^{2\left(t-t^{\prime}\right)}$ and in a finite time the set with $W \geq 1$ is reached. Therefore, $t^{\prime \prime}$ exists and Assumption 1 is valid. In addition, since for $W \geq 1$ the system (9) between jumps has a linear oscillating dynamics with the frequency $\sqrt{2}$, then there is the maximal time between jumps $\tau_{\max }=\sqrt{2} \pi$.

Let $\mathcal{A}=\{\mathbf{x} \in \mathcal{O}:|\mathbf{x}| \leq 1\}$ with $\mathcal{X}_{\mathcal{A}}=\mathcal{O} \backslash \mathcal{A}$ and $\mathcal{U}=\{0\}$ with $\mathcal{X}_{\mathcal{U}}=\{\mathbf{x} \in \mathcal{O}:|\mathbf{x}|<1\}$. Take the Lyapunov function $V(\mathbf{x})=\max \{0, W(\mathbf{x})-1)$, the continuous dynamics $\mathbf{F}$ has the derivative $\dot{V}=0$ for all $\mathbf{x} \in C \cap \mathcal{X}_{\mathcal{A}}$ and $V\left(\mathbf{x}^{+}\right)=\varphi(\sqrt{1+V(\mathbf{x})})^{2}[1+V(\mathbf{x})]-1$ for all $\mathbf{x} \in D \cap \mathcal{X}_{\mathcal{A}}$ (since $W\left(\mathbf{x}^{+}\right)=\varphi(\sqrt{W(\mathbf{x})})^{2} W(\mathbf{x})$ in this case), therefore for all solutions $\phi \in \hat{\mathcal{S}}$ in $\mathcal{X}_{\mathcal{A}}$

$$
\frac{V\left[\phi\left(t_{j+1}, j+1\right)\right]-V\left[\phi\left(t_{j}, j\right)\right]}{t_{j+1}-t_{j}} \leq-\alpha\left(V\left[\phi\left(t_{j+1}, j+1\right)\right]\right),
$$

where $\alpha(s)=\left\{s+1-\varphi(\sqrt{1+s})^{2}[1+s]\right\} / \tau_{\max }$, then all conditions of Theorem 2 are satisfied and the set $\mathcal{A}$ is NZ preasymptotically stable for (11). Choose $U(\mathbf{x})=W(\mathbf{x})$, then the condition (9) is clearly valid for all $\mathbf{x} \in \mathcal{X}_{\mathcal{U}} \cap C$ since $\dot{W}=2 W$, the time domain condition (8) can also be checked for this system, indeed define $W^{j}=W\left[\phi\left(t_{j}, j\right)\right]$, then $(\varphi(|\mathbf{x}|)=1$ for $\left.\mathrm{x} \in \mathcal{X}_{\mathcal{U}}\right)$

$$
\begin{aligned}
W^{j} & =\phi\left(t_{j}, j\right)^{T} \phi\left(t_{j}, j\right) ; \\
W\left[\phi\left(t_{j+1}, j\right)\right] & =W\left[\phi\left(t_{j}, j\right)\right] e^{2\left(t_{j+1}-t_{j}\right)} \\
& =\phi\left(t_{j}, j\right)^{T} e^{\left(\mathbf{A}_{1}^{T}+\mathbf{A}_{1}\right)\left(t_{j+1}-t_{j}\right)} \phi\left(t_{j}, j\right) ; \\
W^{j+1} & =\phi\left(t_{j}, j\right)^{T} \mathbf{P} e^{\left(\mathbf{A}_{1}^{T}+\mathbf{A}_{1}\right)\left(t_{j+1}-t_{j}\right)} \mathbf{P} \phi\left(t_{j}, j\right), \\
\mathbf{P} & =\left[\begin{array}{ccc}
0 & 1 & 0 \\
1 & 0 & 0 \\
0 & 0 & 1
\end{array}\right] .
\end{aligned}
$$

Therefore,

$$
\begin{aligned}
W^{j+1}-W^{j} & =\phi\left(t_{j}, j\right)^{T}\left[\mathbf{P} e^{\left(\mathbf{A}_{1}^{T}+\mathbf{A}_{1}\right)\left(t_{j+1}-t_{j}\right)} \mathbf{P}-\mathbf{I}\right] \phi\left(t_{j}, j\right) \\
& =\phi\left(t_{j}, j\right)^{T}\left[e^{2\left(t_{j+1}-t_{j}\right)}-1\right] \phi\left(t_{j}, j\right) \\
& \geq 2\left(t_{j+1}-t_{j}\right) W^{j}
\end{aligned}
$$

where $\mathbf{I}$ is the identity matrix and the inequality $e^{\eta}-1 \geq \eta$ for $\eta \geq 0$ was used in the last step. Thus all conditions of Theorem 3 (the second part) are verified and the set $\mathcal{U}$ is NZ strictly prerepulsive for the system (11). Finally, all conditions of Theorem 4 are satisfied and the system (11) has an oscillating solution.

The system trajectories for $k=1.5$ are shown in Fig. 3, it has a limit cycle with four jumps. 


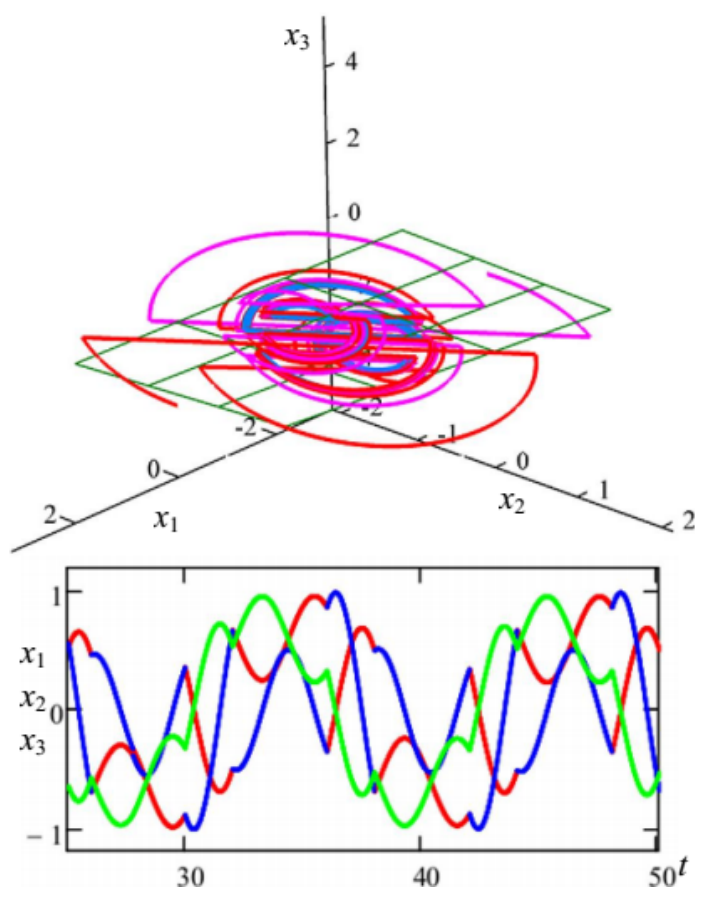

Figure 3. The hybrid system (11) trajectories

\section{A locomotion control for compass-gait biped robot}

The last example is the motivating two-link compass-gait biped robot. In this case the function $\mathbf{G}$ maps the set $D$ to itself, thus formally the system possesses a Zeno-behavior. However, this type of trajectories do not correspond to physical processes in a biped robot, that is why they are neglected and only $\hat{\mathcal{S}}$ is considered. We will be interested in the passive walking, i.e. when $u=0$. In this case the energy

$$
E(\mathbf{x})=0.5 p_{1} x_{2}^{2}-p_{2} \cos \left(x_{1}-x_{3}\right) x_{2} x_{4}+0.5 p_{3} x_{4}^{2}+p_{4}\left(\cos \left(x_{1}\right)-1\right)+p_{5}\left(1-\cos \left(x_{3}\right)\right)
$$

is the system invariant while $\mathrm{x} \in C$ (during the continuous transition phase the system is passive). In the discontinuous phase for $\mathrm{x} \in D$ the energy $E$ can change its value since during the impact the system momentum is preserved only. Moreover, in the vicinity of the stable limit cycle the system can lose or gain the energy during the impact. For the chosen parameters of the system (1) the energy is conserved on the limit cycle.

For this example we will assume that the jump set is defined as $D=\left\{\mathbf{x} \in \mathbb{R}^{4}: \cos \left(x_{1}+\psi+u_{\psi}\right)=\cos \left(x_{3}+\psi+u_{\psi}\right)\right\}$, where $u_{\psi}$ is the slope control from the robot side, i.e. the robot has adjustable ends that can be used to locally advance or postpone the impact (that is equivalent to small variation of the slope angle by a foot regulation). This control is acting during the impacts only by changing the jump map properties. The idea is to use the energy $E$ as a Lyapunov function to determine the stability/instability of the robot. Let $E_{\psi}$ corresponds to the system energy on the limit cycle, then ensuring the energy increasing for $E \leq E_{\psi}$ (the system instability) or its decreasing for $E \geq E_{\psi}$ (stability) by the control $u_{\psi}$ choice we can guarantee the system oscillations (for $E=E_{\psi} \neq 0$ the corresponding trajectories in $C$ are oscillating). It is worth to stress that such a regulation of energy serves as a good example of Theorem 4 application for the system (1), however, the energy stabilization does not imply the robot walking. As we will show in simulation, at least locally this approach leads to the robot locomotion.

By its construction the control $u_{\psi}$ is rather small, thus for its design we can use the first approximation of the system energy after an impact. Let $\phi(t, j)$ be a trajectory of the compass-gait biped robot from $\hat{\mathcal{S}}$, then $E\left(\phi\left(t_{j+1}, j+1\right)\right)=E\left[\mathbf{G}\left(\phi\left(t_{j+1}, j\right)\right)\right]$ and since the map $\mathbf{G}$ depends on $\psi+u_{f}$ let us define $\Sigma\left(\phi\left(t_{j+1}, j\right), \psi+u_{\psi}\right)=E\left[\mathbf{G}\left(\phi\left(t_{j+1}, j\right)\right)\right]$, then

$$
\begin{aligned}
E\left(\phi\left(t_{j+1}, j+1\right)\right) & =\Sigma\left(\phi\left(t_{j+1}, j\right), \psi+u_{\psi}\right) \\
& =\Sigma\left(\phi\left(t_{j+1}, j\right), \psi\right)+\nabla_{\psi} \Sigma\left(\phi\left(t_{j+1}, j\right), \psi\right) u_{\psi}+o\left[u_{\psi}^{2}\right]
\end{aligned}
$$


where $o(\cdot)$ is the Landau notation (for sufficiently small amplitudes of $u_{\psi}$ the term $o\left[u_{\psi}^{2}\right]$ can be neglected). It is assumed that the state vector is available for measurements, then

$$
u_{\psi}(\mathbf{x})=-\varphi_{1}\left[\nabla_{\psi} \Sigma(\mathbf{x}, \psi)\right] \varphi_{2}\left[\Sigma(\mathbf{x}, \psi)-E_{\psi}\right]
$$

is an admissible control strategy with $\varphi_{i}(s) s \geq 0, i=1,2$ for any $s \in \mathbb{R}$. Indeed, introducing the error $\epsilon(t, j)=E(\phi(t, j))-$ $E_{\psi}$ with the substitution of the control (12) into the first approximation we get at the impact:

$$
\begin{aligned}
\epsilon\left(t_{j+1}, j+1\right) & =\epsilon\left(t_{j+1}, j\right)+\nabla_{\psi} \Sigma\left(\phi\left(t_{j+1}, j\right), \psi\right) u_{\psi}\left(\phi\left(t_{j+1}, j\right)\right) \\
& =\epsilon\left(t_{j+1}, j\right)-\nabla_{\psi} \Sigma\left(\phi\left(t_{j+1}, j\right), \psi\right) \varphi_{1}\left[\nabla_{\psi} \Sigma\left(\phi\left(t_{j+1}, j+1\right), \psi\right)\right] \varphi_{2}\left(\epsilon\left(t_{j+1}, j\right)\right),
\end{aligned}
$$

and $\left|\epsilon\left(t_{j+1}, j+1\right)\right|<\left|\epsilon\left(t_{j+1}, j\right)\right|$ for a properly chosen $\varphi_{i}, i=1,2$. The following fact can be formulated.

Corollary 1. For the system (1) with the control (12), there exist $\varepsilon>0, \psi>0, E_{\psi}>0$ and $\varphi_{i}, i=1,2$ such that into the forward invariant set $X=\left\{\mathbf{x} \in \mathbb{R}^{4}:\left|E(\mathbf{x})-E_{\psi}\right| \leq \varepsilon\right\}$ there is an oscillating trajectory.

Proof: Note that $\epsilon(t, j)=\epsilon\left(t_{j}, j\right)$ for all $t \in\left[t_{j}, t_{j+j}\right]$ with $\left(t_{j+1}, j+1\right) \in \operatorname{dom} \phi$, while for a properly selected $\varphi_{i}$, $i=1,2$ the property $\left|\epsilon\left(t_{j+1}, j+1\right)\right|<\left|\epsilon\left(t_{j+1}, j\right)\right|$ is satisfied for $\phi(0,0) \in X$ with a corresponding $\varepsilon>0$ (the first order approximation arguments). Therefore, $X$ is a forward invariant set for (1), (12). Next, there are $\psi>0$ and $E_{\psi}>0$ such that using Theorem 4 it is possible to show that into the set $\left\{\mathbf{x} \in \mathbb{R}^{4}: E(\mathbf{x})=E_{\psi}\right\}$ there is an oscillating trajectory.

The results of the system simulation with $\varphi_{1}(s)=\operatorname{sign}(s)$ and $\varphi_{2}(s)=0.1 \tanh (0.1 s)$ are given in Fig. 4. The initial conditions does not belong to the set of attraction of the limit cycle, and the red dash line at the top of the figure represents the system trajectory without the control. The controlled trajectory is shown by the blue solid line, it converges to the limit cycle. The energy $E$ behavior (it converges to the desired level $E_{\psi}$ ) and the control $u_{\psi}$ are given at the bottom of the figure. The control deviates the slope value during the impact in order to regulate the energy level. During the continuous dynamics phase, the system performs the passive walking (the $E$ is piecewise constant).
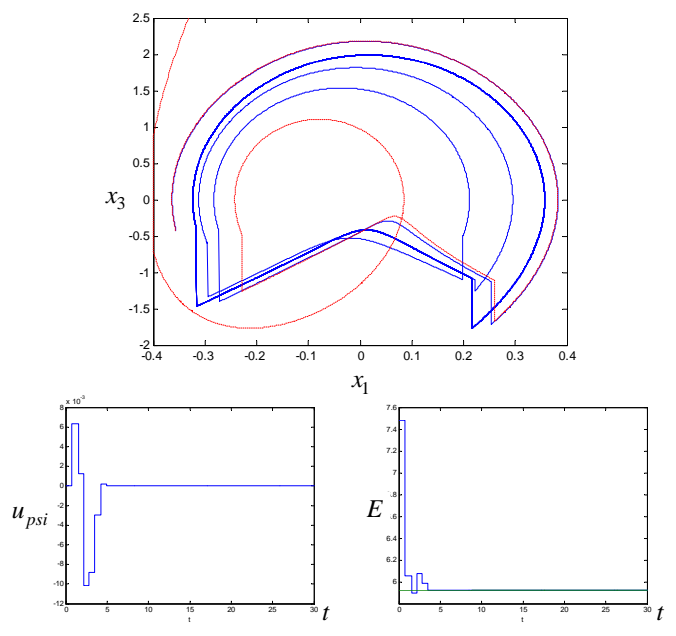

Figure 4. The passive walking with regulated impacts

Finally assume that $D=\left\{\mathbf{x} \in \mathbb{R}^{4}: \cos \left(x_{1}+\psi+\delta_{\psi}(t)+u_{\psi}\right)=\cos \left(x_{3}+\psi+\delta_{\psi}(t)+u_{\psi}\right)\right\}$, where $\delta_{\psi}(t)$ is an unknown bounded time-varying disturbance $\left(\left|\delta_{\psi}(t)\right| \leq 10^{-3}\right.$ for all $t \geq 0$ during the simulation). The results of the same control application are given in Fig. 5. At the top left of the figure the uncontrolled (red dash line) and controlled (blue solid) trajectories are shown, at the top right the slope variation with respect to the reference level is given $\left(\psi(t)=\psi+\delta_{\psi}(t)\right)$, at the bottom the energy and the control are presented.

As we can conclude from the given results of simulation, the proposed control increases the basin of preattraction of the limit cycle and improves the robustness abilities of the robot with respect to the slope variation (the disturbance $\delta_{\psi}(t)$ ). 


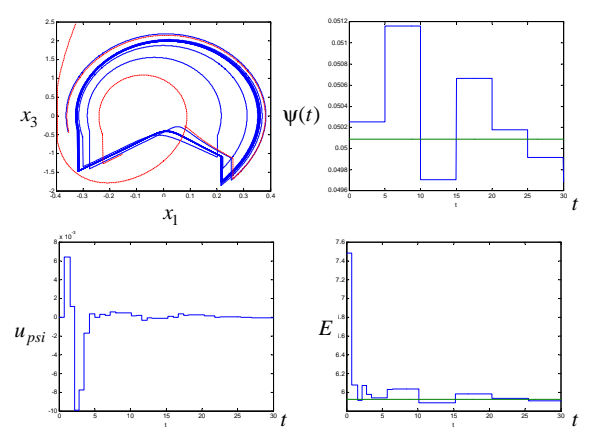

Figure 5. The passive walking with time-varying slope

\section{CONCLUSIONS}

The paper develops the framework of oscillations in the sense of Yakubovich to the class of hybrid systems (having continuous and discrete dynamics). Some sufficient Lyapunov conditions for stability/instability of hybrid systems are presented. In order to facilitate analysis of physical/mechanical systems described by hybrid models without Zeno-behavior, a part of results is formulated for a subset of maximal solutions of the hybrid system. The utility of the arguments is demonstrated on several examples. For the compass-gait biped robot a control strategy is established, which locally stabilizes an unstable limit cycle and ensures locomotion for a time-varying slope of the walking surface. Extensions to different robotic systems are planned in future works.

\section{REFERENCES}

[1] J.-P. Fransoise, Oscillations En Biologie: Analyse Qualitative Et Modèles. Springer-Verlag, August 2005.

[2] G. C. King, Vibrations and Waves. John Wiley \& Sons Inc., August 2009.

[3] J. Kurths, G. V. Osipov, and C. Zhou, Synchronization in Oscillatory Networks. Springer Series in Synergetics, Springer-Verlag, September 2007.

[4] I. Burkin, G. Leonov, and A. Shepelyavyi, Frequency Methods in Oscillation Theory. Mathematics and Its Applications, Dordrecht: Kluwer, 1996. in Russian: 1992.

[5] G. Rogers, Power System Oscillations. The Kluwer International Series in Engineering and Computer Science, Kluwer Academic Pub, 1999.

[6] P. A. Tass, Phase Resetting in Medicine and Biology: Stochastic Modelling and Data Analysis. Springer Series in Synergetics, Springer-Verlag, January 2007.

[7] F. Chernousko and A. Fradkov, eds., Control of Oscillations and Chaos: 2000 2nd International Conference Proceedings. IEEE, November 2000.

[8] A. Fradkov and A. Pogromsky, Introduction to oscillations and chaos. Singapore: World Scientific, 1998.

[9] V. Yakubovich, "Frequency oscillations conditions in nonlinear systems with stationary single nonlinearity," Siberian Math. J., vol. 14, no. 2, 1973.

[10] J. Mallet-Paret and G. Sell, "The poincaré-bendixson theorem for monotone cyclic feedback systems with delay," J. Differential Equations, vol. 125, pp. 441-489, 1996.

[11] V. Yakubovich, "Oscillations in systems with discontinuous and hysteresis nonlinearities," Automation and Remote Control, vol. $12,1975$.

[12] V. Yakubovich and E. Tomberg, "Conditions for self-induced oscillations in nonlinear systems," Siberian Math. J., vol. 30, pp. 641-653, 1989.

[13] D. Efimov and A. Fradkov, "Oscillatority of nonlinear systems with static feedback," SIAM J. Control Optimization, vol. 48, no. 2, pp. 618-640, 2009.

[14] D. Efimov and W. Perruquetti, "Oscillations conditions in homogenous systems," in Proc. IFAC NOLCOS Symp., (Bologna), pp. 1379-1384, 2010.

[15] L. Freidovich and A. Shiriaev, "Transverse linearization for an underactuated compass-like biped robot and analysis of the closed-loop system," in Proc. the 8th IFAC Symposium on Nonlinear Control Systems (NOLCOS), (Bologna), IFAC, August 2010.

[16] B. Morris and J. Grizzle, "Hybrid invariant manifolds in systems with impulse effects with application to periodic locomotion in bipedal robots," IEEE Transaction on Automatic Control, vol. 54, no. 8, pp. 1751-1764, 2009.

[17] A. Shiriaev, L. Freidovich, and S. Gusev, "Computing a transverse linearization for mechanical systems with two and more underactuated degrees of freedom," IEEE Transactions on Automatic Control, vol. 55, no. 4, pp. 893-906, 2010.

[18] A. Shiriaev and C. C. de Wit, "Virtual constraints: a tool for orbital stabilization of nonlinear systems theory," in Proc. 6th IFAC Symposium NOLCOS 2004, (Stuttgart), pp. 1355-1360, IFAC, 2004.

[19] A. Shiriaev and L. Freidovich, "Transverse linearization for hybrid controlled mechanical systems with one passive link," IEEE Transactions on Automatic Control, vol. 54, no. 12, pp. 2882-2888, 2009.

[20] L. T. Aguilar, I. Boiko, L. Fridman, and R. Iriarte, "Generating self-excited oscillations via two-relay controller," IEEE Transactions on Automatic Control, vol. 54, no. 2, pp. 416-420, 2009.

[21] L. Freidovich, U. Mettin, A. Shiriaev, and M. Spong, "A passive 2-DOF walker: Hunting for gaits using virtual holonomic constraints," IEEE Transactions on Robotics, vol. 25, no. 5, pp. 1202-1208, 2009.

[22] C. Cai, A. Teel, and R. Goebel, "Smooth lyapunov functions for hybrid systems part i: Existence is equivalent to robustness," IEEE Trans. Autom. Control, vol. 52, no. 7, pp. 1264-1277, 2007. 
[23] C. Cai, A. Teel, and R. Goebel, "Smooth lyapunov functions for hybrid systems part ii: (pre)asymptotically stable compact sets," IEEE Trans. Aut. Contr., vol. 53, no. 3, pp. 734-748, 2008.

[24] H. Ye, A. Michel, and L. Hou, "Stability theory for hybrid dynamical systems," IEEE Trans. Aut. Contr., vol. 43, no. 4, pp. 461-475, 1998.

[25] F. Forni and A. R. Teel, "Instability and overshoots of solutions for a class of homogeneous hybrid systems by lyapunov-like analysis," in Proc. 49th IEEE Conference on Decision and Control (CDC), (Atlanta), pp. 2390-2395, 2010.

[26] W. Hahn, Stability of Motion. NY: Stability of Motion, 1967.

[27] D. Efimov, "On periodical oscillations of lurie systems with discontinuous nonlinearity," in Proc. 17th IFAC WC, (Seoul, Korea), pp. 5927-5932, 2008. 